\title{
Autoconcepto y motivación en adolescentes que abandonan voluntariamente sus estudios de preparatoria. Una aproximación cualitativa
}

\section{The influence of self-concept and motivation in adolescents who voluntarily dropout from highschool. A cualitative approach}

\author{
María Inés Gómez del Campo del Paso (iD \\ $\triangle$ igomezdelcampo@hotmail.com \\ Universidad Michoacana de San Nicolás de Hidalgo, Morelia, México.

\section{Belem Medina-Pacheco (D)} \\ $\bowtie$ belemed@yahoo.es \\ Universidad Michoacana de San Nicolás de Hidalgo, Morelia, México.
}

Recibido: $30-04-2018$

Iván Hernández-Martínez (ic)

Universidad Michoacana de San Nicolás de Hidalgo, Morelia, México.

Aceptado: 10-09-2018

Publicado: 01-10-2018

\section{RESUMEN}

Objetivo. Describir el autoconcepto y la motivación de un grupo de adolescentes de la ciudad de Morelia, Michoacán, con edades de dieciséis a diecinueve años, que abandonaron sus estudios de educación media superior. Método. Para indagar estos aspectos, se realizaron entrevistas a seis adolescentes -tres mujeres y tres hombresque dejaron la preparatoria inconclusa. Los resultados se presentan organizados en cuatro categorías: autoconcepto, autoestima, motivación y aplazamiento contingente. Resultados. Se destaca que los adolescentes entrevistados ponen énfasis en los aspectos negativos de su persona, tienden a posponer las cosas y en general vivían la escuela como un obstáculo o barrera para poder obtener metas más importantes para ellos, como lograr independencia y hacer otras actividades. Conclusiones. En muchos casos la deserción fue impulsiva, sin embargo, los estudios no se han retomado por falta de decisión. Se considera que profundizar en este tema permitirá ofrecer a los adolescentes acompañamiento durante la preparatoria y una mejor orientación vocacional para que puedan hacer elecciones más conscientes y reflexivas.

Palabras clave: Deserción escolar, adolescente, autoconcepto, motivación, aplazamiento contingente. 


\section{ABSTRACT}

Objective. To describe the self-concept and motivation of a group of adolescents from the city of Morelia, Michoacán, aged 16 to 19, who dropped out of high school. Method. Interviews were conducted with six adolescents -three women and three men- who left the unfinished high school to investigate these aspects. The results are organized into four categories: self-concept, self-esteem, motivation and contingent deferment. Results. The adolescents interviewed put emphasis on the negative aspects of their person, they tend to postpone things, and in general lived the school as an obstacle or barrier to obtain more important goals for them, such as achieving independence and doing other activities. Conclusions. In many cases, the desertion was impulsive, however the studies have not been resumed due to lack of decision. It is considered that deepening in this theme will offer young people support during high school and better vocational guidance, so that they can make more conscious and reflective choices.

Keywords: Dropout, adolescent, self-concept, motivation, contingent deferment.

\section{INTRODUCCIÓN}

Según la Organización para la Cooperación y el Desarrollo Económicos (OCDE) (2014), en México las tasas de matriculación continúan bajas en el rango de quince a diecinueve años de edad; la deserción escolar se presenta como una de las principales razones que encabezan este panorama. La reforma educativa hizo obligatoria la educación media superior a partir del 2012. Dicha reforma tiene por objetivo la cobertura total en este nivel para 2022, como un paso importante para reducir los altos niveles de deserción en el país. No obstante, muchos adolescentes dejan sus estudios, sin que exista un motivo académico, como la reprobación o expulsión, ni una necesidad económica en casa que los obligue a abandonar la escuela.

Por este motivo es que se considera importante describir el autoconcepto y la motivación de estos adolescentes que dejaron sus estudios. También es importante examinar si una vez que abandonan los estudios tienen la intención de continuarlos o conocer cómo visualizan su futuro. A partir del estudio se pueden proponer intervenciones psicológicas individuales o colectivas para apoyar a los adolescentes de manera que retomen sus estudios o para que tengan un plan de vida claro que no incluya más estudios.

La deserción escolar suele presentarse acompañada de una serie de factores contextuales; para conocer esta situación se considera importante la comprensión de procesos psicosociales, motivacionales y personales. Resulta frecuente que las razones más comunes para el abandono escolar tengan que ver con las dificultades socioeconómicas: falta de recursos económicos, problemas familiares (poco apoyo familiar, conflictos con los padres, hermanos) y problemas académicos (bajo nivel de aprovechamiento, expulsión escolar, falta de matrícula universitaria), entre otras (Instituto Nacional de Estadística, Geografía e Informática, 2015).

El tema de la deserción escolar en la educación media superior en nuestro país ha sido ampliamente estudiando. Por ejemplo, Abril, Román, Cubillas y Moreno (2008) presentaron un análisis de las causas de la deserción en la educación media superior en el estado de Sonora. Estas autoras identifican cinco causas: factores económicos, falta de oferta educativa, problemas familiares, falta de interés y problemas académicos. Encontraron que el $26 \%$ de los estudiantes de la muestra de estudiantes que había abandonado sus estudios lo hizo por falta de interés, estas autoras consideran que puede 
relacionarse con falta de metas y proyecto de vida en los adolescentes, sin embargo, no indagan más a fondo. En este caso se trataba de adolescentes que consideraban su desempeño en la escuela como regular y quienes, a pesar de que en su mayoría se manifestaron en desacuerdo con el nivel educativo alcanzado, no tenían planes concretos de continuar sus estudios.

Por su parte, Ruiz-Ramírez, García-Cué y Pérez-Olvera en 2014, en el artículo titulado "Causas y consecuencias de la deserción escolar en el bachillerato", investigaron a un grupo de dieciocho desertoras y diecisiete desertores, cuatro años después de haber abandonado sus estudios de preparatoria en la Universidad Autónoma de Sinaloa. El objetivo fue establecer las causas y consecuencias de tipo personal, económico y social que provocaron la deserción escolar de los participantes. Esta investigación se realizó con una metodología de enfoque mixto. Los resultados indicaron que el principal factor asociado a la deserción escolar fue el personal, y se destacaron como causas casarse y no aprobar materias. La principal consecuencia del abandono de los estudios fue de tipo económico.

También, Sánchez (2015) exploró las causas de la deserción en la educación media superior del bachillerato de la Universidad Tecnológica de la ciudad de León, Guanajuato. En este estudio se encontró que la principal causa de deserción es la reprobación de varias materias; otras causas fueron falta de apoyo escolar, falta de motivación y el desempeño docente. Esto corrobora el estudio de Landero (2012), quien destaca como causas principales de la deserción escolar en estudiantes entre quince y diecisiete años los problemas económicos y la reprobación de materias.

Por otro lado, en el estudio de caso realizado por Peña, Soto y Calderón (2016), con estudiantes de secundaria de dos instituciones de las comunas de Padre de las Casas y Villarrica, Región de la Araucanía, Chile, se encontró que el entorno familiar es más determinante que el factor económico para la deserción escolar en adolescentes de entre catorce y diecisiete años, pertenecientes a estos liceos. Destaca la falta de apoyo de los adultos responsables respecto a la educación de los adolescentes como causa de deserción.

Como se puede observar, en estos estudios se destacan diferentes causas, sin embargo, en el presente estudio se decidió enfatizar en los factores internos, como son la motivación y el autoconcepto, por lo que a continuación se presentan algunas consideraciones sobre estos constructos.

El autoconcepto en el adolescente y su relación con el desempeño escolar. Diversos autores e investigadores han estudiado el autoconcepto y lo definen finalmente como aquello que la persona es realmente, la forma como se muestra a los demás, lo que quiere lograr o llegar a ser. Considera el aspecto físico, las relaciones, las propiedades, las creencias y los valores (González-Pineda, Núñez-Pérez, González-Pumariega y García García, 1997; Jourard y Landsman, 1998; Sneed y Whitbourne, 2003; Denegri, Opazo y Martínez, 2007; Penagos, Rodríguez y Carrillo, 2009).

De acuerdo con Correa Romero, Saldívar Garduño y López Suárez (2015), la relación entre el autoconcepto académico y los logros escolares ha sido ampliamente estudiada, hasta el punto de que ha quedado demostrado que este aspecto tiene un efecto inmediato en el rendimiento escolar, ya que aumenta la confianza y la motivación, y mejora el desempeño de los estudiantes. Sin embargo, en esta ocasión se pretende abordar el autoconcepto como un factor que dificulta la continuación de los estudios o la reanudación de estos una vez interrumpidos. 
Por otra parte, Gómez del Campo, Salazar y Rodríguez (2013) establecen que un autoconcepto adecuado es un elemento fundamental para que el joven pueda adquirir las competencias sociales que requiere en la universidad, ya que un autoconcepto positivo permite que la persona se sienta más competente, lo cual facilita la responsabilidad en la toma de decisiones. Esto mismo se muestra en el estudio de Lila, García, García y Fuentes (2011), cuyos resultados indicaron que un mejor autoconcepto se corresponde con el mejor ajuste psicológico, una buena competencia personal y menos problemas comportamentales. Por su parte, Tapia, Fiorentino y Correché (2003), quienes encontraron que en los adolescentes con mejor autoconcepto presentaban bajo nivel de aislamiento y soledad.

La escuela puede ejercer influencia en la formación del autoconcepto, y otorgar al adolescente recursos como el reconocimiento de sí mismo, así como apoyo emocional y afectivo. De acuerdo con Woolfolk (2006), en la escuela, el autoconcepto influye en el aprendizaje a través de la selección de recursos. Rogers (1980) menciona que probablemente la escuela es la institución más influyente para los adolescentes, por encima de la familia, iglesia, policía y al Gobierno, en lo que se refiere al modelo de la política interpersonal de la persona en crecimiento.

La motivación hacia los estudios. Durante la adolescencia, los padres y la sociedad en general esperan algo del joven y con el tiempo este hecho se volverá más presente en èl. También es frecuente que los adolescentes comenten entre ellos las ocupaciones que ejercerán en un futuro posterior a la graduación; hay veces que la presión social llega de tal manera que los familiares del adolescente constantemente le enfatizan la necesidad de continuar con los estudios (Muñoz Izquierdo y Guzmán, 2010).

De acuerdo con Horrocks (2012), existen otras presiones que vive el adolescente, como también situaciones que dan pie a que piense cada vez más en su ocupación profesional y con ello en la planificación de su futura vida; una de estas proviene del deseo que tiene el adolescente de poder llegar a experimentar una libertad personal e independencia económica, también pensar sobre el matrimonio y las implicaciones que este conlleva. Algunos adolescentes no siguen este patrón típico, sin embargo, la mayoría se ajustará en mayor o menor grado a este patrón.

Según Hernández, Rodríguez y Vargas (2012), la motivación es crucial para que un estudiante tenga interés en lo que aprende y logre satisfacción en lo que hace. Los factores sociales influyen en este aspecto, sin embargo, es fundamental que el adolescente cuente con motivación intrínseca, ya que esta le permite sentirse capaz, confiar en sí mismo y considerar que el estudio puede mejorar su calidad de vida.

El presente estudio se centra exclusivamente en el abandono voluntario de la escuela, ya que esta causa se vincula directamente con el individuo y está dentro del control de este. Abandonar los estudios de bachillerato o preparatoria por falta de interés tiene que ver con aspectos que están más allá de las condiciones de su entorno y dificultades para estudiar, y se suscriben más a los aspectos motivacionales y del autoconcepto del estudiante. Por lo tanto, se considera importante investigar qué piensan estos adolescentes respecto a su autoconcepto y si consideran que influyó en su decisión de abandonar los estudios y en su falta de motivación hacia el estudio y la escuela.

El objetivo de la presente investigación fue describir el autoconcepto y la motivación de un grupo de adolescentes de la ciudad de Morelia, Michoacán, con edades entre dieciséis y diecinueve años, que abandonaron sus estudios de educación media superior. Así de esta forma: Identificar el autoconcepto de los adolescentes y la forma en que este influye en las decisiones que toman. Explorar la motivación de los adolescentes tanto antes como después de dejar los estudios. Explorar los planes futuros de este grupo de adolescentes. 


\section{MÉTODO}

Esta investigación se realizó con un enfoque cualitativo, desde una perspectiva fenomenológica, ya que se exploró la percepción que los adolescentes que abandonan sus estudios tienen sobre sí mismos, con énfasis en su experiencia personal, en lugar de buscar características o motivos generales (Álvarez-Gayou, 2009; Martínez, 2013; Rodríguez, Gil y García, 1999). Se consideró interesante abordar el problema de la deserción escolar desde esta perspectiva, para darles voz a los involucrados en la situación y conocer los motivos que ellos consideran relevantes, además de su autoconcepto y planes futuros respecto a su educación. La técnica utilizada fue la entrevista semiestructurada, que de acuerdo con Guerrero-Castañeda y Ojeda-Vargas (2014) constituye una de las técnicas que se utiliza con frecuencia en la investigación fenomenológica, en la cual, aunque el entrevistador utiliza un guion, decide dónde poner énfasis e incluso puede incluir preguntas de acuerdo a la forma como se va realizando la entrevista.

Se entrevistó a tres hombres y tres mujeres, de acuerdo a los siguientes criterios de inclusión: tener entre dieciséis y veinte años, haber abandonado los estudios de nivel medio superior por decisión propia y estar dispuesto a participar. Como criterios de exclusión se consideró el hecho de abandonar los estudios por embarazo, problemas económicos o reprobación.

La investigación se realizó a través de entrevistas semiestructuradas sobre cuatro dimensiones: social, familiar, personal y escolar. Las preguntas guía abarcaron cada una de las dimensiones de interés, lo que permitió profundizar lo suficiente para hacer el análisis. Las entrevistas fueron grabadas y posteriormente transcritas para su análisis. Consideraciones éticas: todos los participantes de la investigación fueron voluntarios, se asignaron claves a los participantes para evitar que fueran reconocidos. F1, F2 y F3 para las mujeres y $\mathrm{H} 1, \mathrm{H} 2$ y $\mathrm{H} 3$ para los hombres. Debido a que este artículo toma como base una tesis de licenciatura, fue autorizado por el Departamento de Titulación que funge como Comité de Ética por parte de la Facultad de Psicología de la Universidad Michoacana de San Nicolás de Hidalgo (UMSNH).

\section{RESULTADOS}

Los resultados se organizaron en tres categorías: autoconcepto, motivación y también se decidió incluir la categoría de aplazamiento contingente, ya que apareció en todos los participantes. A continuación, se presentan las definiciones de cada categoría, así como fragmentos de los discursos de los participantes respecto a esos temas y su comparación con los estudios y teorías consultados.

Categoría autoconcepto. La principal importancia de explorar el autoconcepto en los participantes era comprender la manera en que ellos se percibían y evaluaban, así como también conocer la relación que tienen con su situación escolar. Se encontró que el nivel de conocimiento que los participantes tienen de sí mismos se relaciona con la capacidad para conocer aspectos relacionados con el progreso y retroceso de su potencial, lo que incluye la situación escolar.

Al realizar una descripción de sí mismos, se pueden apreciar los elementos esperados en la descripción del autoconcepto; como se ven cuando se miran a sí mismos en términos de sus características físicas auto percibidas, de su personalidad, sus habilidades, sus rasgos, sus roles y su estatus social (Denegri, Opazo y Martínez, 2007; Penagos, Rodríguez y Carrillo, 2009). 


\section{Testimonios}

F1: Soy muy penosa, hiperactiva, con mis amigos, con mi familia igual, je je je [risas]. F2: Creo que soy una persona muy vulnerable, sensible. A mí me gusta ser muy sociable y me considero muy buena persona, y pues también me gusta dar consejos [risas]. Y ¿lo que no me gusta de mí? pues es que a veces soy muy impulsiva. Me enojo, como que por una cosa ya me enojé y como que me gusta desquitarme mi coraje.

F3: Mira lo que más me gusta de mí es que cuando me propongo algo lo logro, lo que no me gusta de mí es que lo dejo a la desidia; soy entusiasta pero lo que no me gusta es que soy muy explosiva; mi estatura no me gusta; me gusta mi sonrisa.

H1: Soy determinado, feliz y como estoy viviendo ahorita, mi independencia. No me gusta que no esté estudiando la carrera, la impuntualidad, holgazán e informal.

H2: Me gusta que cuando me propongo algo, siempre lo logro. Me gusta también que soy atento y trabajador. Me disgusta que dicen que soy bien güevon [flojo], agresivo, que no puedo controlarme. Rencoroso, me desquito en el momento.

H3: Carismático, trato de ser feliz siempre con optimismo [...] nervioso, apresurado y por eso tengo malos resultados y me dejo llevar por la ira. Cariñoso, intolerante e inteligente. Tengo un temperamento indeciso.

Se puede apreciar que los aspectos que más les gustaban a los participantes fueron: la consecución de las metas, el desempeño, la sociabilidad, la independencia, aspectos de carisma y bienestar. Por otra parte, aquellos aspectos que más disgustaron fueron: no estudiar, ser informal (impuntualidad, desidia, "flojera"), impulsividad, rencor, agresión, nerviosismo y la estatura. Es importante mencionar que se les facilitaba identificar aquellas características que les disgustaban de su persona y que les daban más valor a estos aspectos que a los aspectos que les agradaban de sí mismos. Aunque existía una contradicción entre algunas características, parece ser que en ciertos aspectos de su vida se sentían más competentes que en otros, en algunas situaciones son capaces de lograr sus metas y están satisfechos con su desempeño, pero en otros no.

El manejo inadecuado del enojo y la conducta impulsiva parecen estar implicados en las decisiones que los adolescentes tomaban. La mayoría de los participantes, al momento del abandono escolar, no contaba con los recursos necesarios para administrar estas emociones y lidiar con las mismas. La salida más común por la que optaban era abandonar la escuela. Se puede encontrar una relación entre el manejo inadecuado del enojo y la impulsividad con una toma de decisiones apresurada y emocional, más que racional.

Estos resultados coinciden tanto con lo encontrado tanto por Correa Romero, Saldívar Garduño y López Suárez (2015), como por Lila, García, García y Fuentes (2011) respecto a que los sentimientos hacia los estudios influyen en el autoconcepto que tiene el estudiante respecto de sí mismo. Los adolescentes entrevistados no consideraban tener las habilidades necesarias para ser buenos estudiantes, o bien algunas de las características que tienen les dificultaron su desempeño escolar. Otro aspecto importante que se encontró al entrevistar a los adolescentes fue el de los sentimientos de desvaloración; al momento de la deserción escolar los adolescentes se sentían inadecuados y poco conformes con ellos mismos, lo cual influyó definitivamente en su decisión de abandonar la escuela:

\section{Testimonios}

F2: Sí me sentía muy estresada, creía que no valía nada por que como que todos estaban en otro lugar y yo era la única persona que estaba ahí, ¿si me entiendes? o sea como que yo me sentía mucho muy sola y no había nadie que me apoyara en ese momento.

H3: Yo no me veía igual a mis amigos porque ellos sí estaban entrando a sus carreras y yo no, yo veía que me iba quedando atrás. 
Lo anterior coincide con los hallazgos de Tapia, Fiorentino y Correché (2003) y de Gómez del Campo, Salazar y Rodríguez (2014) respecto a que un adecuado autoconcepto repercute en mejores habilidades sociales y disminuye el aislamiento.

Categoría motivación. Cuando se les preguntó sobre las principales aspiraciones académicas y laborales que tenían mientras estaban estudiando contestaron lo siguiente:

\section{Testimonios}

F1: Estudiar, tener una licenciatura, enfermería.

F2: Quería ser doctora.

F3: Mira, en académicas quería terminar mi carrera, que sería bióloga.

H1: Ventas. Como el comercio. El estudio no me interesaba mucho.

H2: Pues todavía quería ser piloto aviador. Quería entrar a una escuela militar, nada más que lo dejé al último.

H3: Ingeniería mecánica.

Es decir que, salvo en un caso, la intención de los participantes era concluir una carrera universitaria, por lo que terminar la preparatoria era un paso indispensable. Sin embargo, cuando se les pidió que dijeran cuáles habían sido los motivos y las razones por las cuales no continuaron estudiando dijeron:

\section{Testimonios}

F1: Ya no quise la verdad, ya me había aburrido la escuela. Es que soy muy penosa, hubo problemas, fueron problemas de salud, y que repercutieron.

F2: Porque... pues ya no me gustaba y pues me sali porque ya no me gustaba ya no quise seguir estudiando... O sea, no me gustaba porque a veces como que me enfadaba, de estar escribiendo o los maestros y cuando me di cuenta de que para ser doctora eran como cinco años dije: no, ya me truncó y no quiero seguir.

F3: A veces pierdo el interés y dejo a la desidia ciertas cosas, mis ideas son terminar de estudiar, [...] también a veces pienso que no, ¿para qué estudiar si de todos modos voy a trabajar?, pero ya luego me pongo a pensar que sería más fácil si estudiara, tendría como la vida más fácil [...] es mejor estudiar que trabajar.

H1: Me aburría el lugar donde estaba, faltaba mucho y prefería hacer otras cosas en lugar de andar ahí en la escuela. O sea, pensaba hacer la carrera, pero no ejercerla, así como por libertinaje, pero nada más.

H2: Pues es que yo no siempre podía estar con mis amigos, dejé de estudiar porque quería salir más con mis amigos.

H3: Quería descansar y además tenía muchas distracciones en mi casa. Por eso preferí dejar la escuela.

Durante la entrevista se pudo notar la "aceptación" que los participantes tenían de su condición como estudiantes en rezago, y que preferían dedicarse al trabajo, convivir con los amigos, las distracciones, entre otros. Sin embargo, esta aceptación parece anteponerse con los sentimientos que expresaban ante su deserción escolar; es posible que las condiciones externas se hayan asimilado de tal modo que ellos consideran que su decisión fue únicamente personal, sin diferenciar con precisión si existieron influencias externas.

Se puede considerar que los deseos de libertad e independencia de estos adolescentes se expresaron en dejar de lado ciertas obligaciones y responsabilidades. Por lo que se puede apreciar que la escuela no era en sí misma un motivador suficiente, por lo que pesaron más sus deseos e intereses. Sin embargo, los adolescentes sabían que se esperaba algo de ellos, por lo que hicieron suyas unas metas que muy posiblemente no eran propias, lo que provocó, tal como explican Hernández, Rodríguez y Vargas (2012), que al presentarse dificultades no tuvieran la motivación suficiente para sobreponerse a ellas y continuar estudiando. 
Categoría del aplazamiento contingente. Esta categoría surge por el patrón constante que mostraron todos los participantes cuando hablaban de su futuro. Se encontró que existía una tendencia implícita y explicita para aplazar la prosecución escolar; ocurrieron distintos momentos del discurso en los que por iniciativa propia decían querer volver a estudiar: "ahorita no", "ya", "después", entre otras. Estos momentos específicos del discurso que se presentaron durante la entrevista habilitaron al concepto con un aspecto de contingente, como sinónimo de "incierto", "aleatorio", "eventual" o "posible", pero no "seguro", al menos desde la propia perspectiva del participante. También resulto interesante la tendencia para aplazar los estudios; todos los participantes tenían el deseo de continuar en un futuro, sin embargo resultaba difuso en qué tiempo exactamente los reanudarían.

Distintos momentos del discurso de los participantes sirven para ilustrar la presencia de esta categoría; sin embargo, con la finalidad de conocer la manera en que los adolescentes se proyectaban hacia el futuro, se les pidió que dijeran cómo visualizaban su futuro dentro de tres años. Esto fue lo que contestaron:

\section{Testimonios}

F1: Pues ya yo creo estudiando, pues si yo digo nunca es tarde. El año que entra ya ja ja ja [risas]

F2: ¿Cómo, mi futuro? pues no sé... como que... ja [risa] pues no sé... espero que, pues sí pienso meterme a estudiar ahorita, pero no sé cuándo, a lo mejor cuando ya esté lista o cuando ya piense que si va a estar mejor. Sí quiero meterme, pero no sé, creo que ahorita todavía no.

F3: Pues mira, sí me arrepiento de no haber estudiado porque sé que la situación está difícil ahorita, sí se puede, sí quisiera estudiar, sí se puede, pero también es como que falta de decisión, de decir "sí puedo", sería como inseguridad más que nada.

H1: Sí quiero seguir estudiando. Ya tener la prepa hecha [risas], un trabajo más o menos estable, durar un año mínimo en un trabajo, ya que no duro ni cuatro meses en uno; no me veo casado, con hijos y me veo otra vez viviendo en Morelia.

H2: Tengo pensado meterme a una escuela militar, así ya directamente ya me veo, ya... pasando mis exámenes para la carrera de piloto, no es aquí, ya me veo estudiando para poder llegar a esa carrera, así en tres años.

H3: En la escuela, universidad y más independiente de mis padres.

La presencia de un aplazamiento contingente para la prosecución escolar puede deberse, en gran medida, a la importancia que para los adolescentes tiene la imagen del éxito, así como a una visión del futuro cimentada en sus talentos, habilidades, esfuerzo personal y escolaridad. De manera que, si fuera el caso, un estudiante que no tenga la capacidad para satisfacer de alguna forma sus aspiraciones académicas y laborales, en especial los que tengan menos de veinticinco años de edad, tendrán por lo general destruida su imagen de éxito y su apreciación positiva del futuro (Pérez, Samper y Mestre, 2001). Es por eso que los adolescentes no descartan la continuación de sus estudios de bachillerato, aunque los ubican en un futuro no determinado y de hecho no realizan intentos formales de reanudarlos.

\section{CONCLUSIONES}

La presente investigación permitió conocer cómo influyen la autoestima, el autoconcepto y la motivación en los adolescentes de la ciudad de Morelia que se encuentran en deserción escolar. De acuerdo con los datos encontrados y el análisis realizado se concluye lo siguiente: la deserción escolar, en las condiciones que el grupo presenta, se manifiesta cuando el adolescente pierde el interés de continuar con los estudios y 
piensa constantemente en su propia independencia. Un aspecto que no se evidencia en el presente trabajo, y sin embargo podría ser un elemento importante, es la forma como el grupo de pares influye para la decisión de abandonar los estudios o continuarlos; solo uno de los participantes comentó sentir que sus amigos "lo dejaban atrás". Esta presión tenía una importante implicación en la manera de sentirse y evaluarse en la mayoría de los participantes, mientras que la institución escolar no pareció tener un papel relevante en la conformación del autoconcepto y la autoestima.

Se observó que los participantes tenían un autoconcepto claro al momento de abandonar sus estudios, pero enfatizaron sus características negativas y tenían en general una sensación de no ser competentes. Se observa que los adolescentes no se sentían capaces de cumplir con las expectativas que tenían respecto a sí mismos; es decir que su yo ideal no correspondía a sus logros reales y esto les generó tristeza, impotencia y apatía. Al empezar a trabajar esta sensación de fracaso desaparece, por lo tanto, su autoestima mejora.

Un aspecto importante a considerar es que la decisión de desertar de la escuela se presenta en un momento en que existen sentimientos de inadecuación, desvalorización y falta de apoyo. Esto, aunado a la impulsividad, pudo haber favorecido el abandono de la escuela. En este sentido, sería importante planear intervenciones con los adolescentes en los momentos que se sienten poco capaces para reforzar sus habilidades y hacerlos conscientes de sus metas, lo cual favorecerá que continúen sus estudios en lugar de desertar.

Existe la tendencia generalizada entre los participantes de aplazar la reanudación escolar. La falta de claridad en sus discursos, respecto a continuar sus estudios, habilita al concepto de un aspecto contingente y confirma la importancia que para ellos tiene la imagen del éxito y visión del futuro. Una de las metas centrales en todos los participantes es continuar con los estudios, ejercer una profesión y ser personas más independientes. Sin embargo, no existen evidencias reales de acciones concretas para continuar sus estudios, por lo que sería importante trabajar con ellos sus planes de vida para que tengan metas propias y las puedan llevar a cabo.

Los hallazgos muestran la posibilidad de relacionar el autoconcepto con la deserción escolar, esta relación mantiene una influencia significativa con la manera en que se presenta crecimiento y desarrollo personal en los adolescentes que abandonan sus estudios. Un aspecto importante para reflexionar es la necesidad de que los adolescentes consideren los estudios de preparatoria como un elemento fundamental para el logro de éxitos futuros, ya que si no son capaces de hacer esta vinculación, será más probable que abandonen sus estudios.

Se enfatiza la importancia de profundizar en otros conceptos revisados: familia, contexto social y escolar, así como en la atención a este sector juvenil. Los participantes tienen suficientes potencialidades para continuar con sus estudios, sin embargo hace falta que dicho sector cuente con programas que orienten al adolescente hacia la expresión y el manejo de las emociones, así como de orientación vocacional.

En futuras investigaciones podría ser útil explorar otros conflictos que se complican a partir de la deserción escolar (depresión, abuso de substancias, conductas disruptivas) así como también en áreas que sean importantes para el propio adolescente.

Es también conveniente explorar las historias familiares, descritas desde los mismos miembros de la familia, y extraer información útil del entorno del participante. La participación de otros grupos, con características distintas o similares se considera muy útil para delimitar las variables y comparar los resultados. 


\section{REFERENCIAS}

Abril, E.; Román, R.; Cubillas, M.J. y Moreno, I. (2008). ¿Deserción o autoexclusión? Un análisis de las causas de abandono escolar en estudiantes de educación media superior en Sonora. Revista Electrónica de Investigación Educativa, 10(1), 1-16.

Álvarez-Gayou J.L. (2009). Cómo hacer investigación cualitativa: fundamentos y metodología. México: Paidós Mexicana.

Correa Romero, F.; Saldívar Garduño, A. y López Suarez, A. (2015). Autoconcepto y estados emocionales: su relación con la motivación en adolescentes. Enseñanza e investigación en psicología, 20(2).

Denegri, C.M.; Opazo P.C y Martínez, T.G. (2007). Aprendizaje cooperativo y desarrollo del autoconcepto en estudiantes chilenos. Revista de Pedagogía, 28(81),13-41.

Gómez del Campo, M.I.; Salazar, M.L. y Rodríguez, E.I. (2014). Los talleres vivenciales con enfoque centrado en la persona, un espacio para el aprendizaje de competencias sociales. Revista intercontinental de psicología y educación, 16(1), 175-190.

González-Pineda, J.A.; Núñez-Pérez, J.C.; González-Pumariega, S. y García García, S. (1997). Autoconcepto, autoestima y rendimiento escolar. Revista Psicothema, 9(2), 271-289.

Guerrero-Castañeda, R.F. y Ojeda-Vargas, M.G. (2015). La fenomenología, y su uso en la producción científica de Enfermería: estudio bibliométrico 2010-2014. Ra Ximhai, 11(2), 193-206.

Hernández, H.C.A.; Rodríguez, P.N. y Vargas, G.A.E. (2012). Los hábitos de estudio y motivación para el aprendizaje de los alumnos en tres carreras de ingeniería en un tecnológico federal de la ciudad de México. Revista de la educación superior, 41(163), 67-87, S0185-27602012000300003.

Horrocks, J.E. (2012). Psicología de la adolescencia. México: Trillas.

Instituto Nacional de Estadística, Geografía e Informática (INEGI). (2015). Principales resultados de la Encuesta Intercensal 2015. Estados Unidos Mexicanos: INEGI.

Jourard, S.M. y Landsman T. (1998). La personalidad saludable: El punto de vista de la psicología humanística. México: Trillas.

Landero, J.F. (2012). Deserción en la educación media superior en México. Suma por la educación, 2-85. Recuperado de: editor.pbsiar.com/upload/PDF/desercion.pdf

Lila, M; Gracia, E; García, J.F.; Fuentes, M C; (2011). Autoconcepto y ajuste psicosocial en la adolescencia. Psicothema, 23. 7-12.

Muñoz Izquierdo, C. y Guzmán, J.T. (2010). Una exploración de los factores determinantes del rendimiento escolar en la educación primaria. Revista Latinoamericana de Estudios Educativos (México), XL(2), 167-191. 
Organización para la Cooperación y el Desarrollo Económicos (OCDE). (2014) Panorama de la educación 2014, México: OCDE. Recuperado de: https://www.oecd.org/edu/ Mexico-EAG2014-Country-Note-spanish.pdf

Penagos, A.; Rodríguez, M. y Carrillo, S. (2009). Apego, relaciones románticas y autoconcepto en adolescentes bogotanos. Revista de la Pontificia Universidad Javeriana, 5(1) 21-36.

Peña, J.C.; Soto, V. y Calderón, U. (2016). La influencia de la familia en la deserción escolar. Estudio de caso. RMIE SCIELO, 21(70), 881-899.

Pérez, E.; Samper, P. y Mestre, V. (2001). Clima familiar y desarrollo del autoconcepto. Un estudio longitudinal en población adolescente. Revista Latinoamericana de Psicología, 33, 243-259, id=80533301.

Rodríguez, G.; Gil, J. y García, E. (1999). Metodología de la investigación cualitativa. España: Aljibe.

Rogers, C. (1980). El poder de la persona. México: El manual moderno.

Ruiz-Ramírez, R.; García-Cué, J.L. y Pérez-Olvera, M.A. (2014). Causas y consecuencias de la deserción escolar en el bachillerato: caso Universidad Autónoma de Sinaloa. Revista Ra Ximhai, Universidad Autónoma Indígena de México, 10(5), 51-74, id $=46132134004$.

Sánchez, S.E. (2015). La reprobación, principal factor que origina la deserción escolar en la educación media superior en León, Gto. Reaxion Ciencia y Tecnología Universitaria, 77. Recuperado de: reaxion.utleon.edu.mx

Sneed, J.R. y Whitbourne, S.K. (2003). Identity Processing and Self-Consciousness in Middle and Later Adulthood. Journal of Gerontology: psychological sciences, 58B (6), 313-319.

Tapia, M.L.; Fiorentino, M.T. y Correché, M.S. (2003). Soledad y tendencia al aislamiento en estudiantes adolescentes. Su relación con el autoconcepto. Fundamentos en Humanidades, IV, 163-172, id=18400809.

Woolfolk, A. (2006) Psicología educativa. México: Pearson Educación. 